\title{
El niño con hipertransaminasemia: ¿cómo continuar?
}

\section{The child with hypertransaminasemia: what's next?}

\author{
Dr. Alejandro Costaguta $a^{a}$ Dr. Fernando Álvarez ${ }^{b}$
}

\section{RESUMEN}

El aumento de las cifras de transaminasas séricas puede observarse en diferentes escenarios de la consulta médica cada vez con mayor frecuencia. Esto supone un desafío complejo para el pediatra dada la escasa información bibliográfica que existe sobre este tema. Desde el paciente completamente asintomático hasta el niño con signos de cronicidad, el espectro es muy variado y obliga a orientar rápida y eficientemente la investigación a fin de evitar costos y sufrimientos innecesarios para el paciente y su familia. El trabajo coordinado con el hepatólogo es recomendable en la mayoría de los casos.

Palabras clave: transaminasas, hipertransaminasemia, niños.

\section{ABSTRACT \\ The raising of serum transaminases is seen with increasing frequency in different scenarios from daily practice. This poses a complex challenge to the pediatrician owing to the scarcity of bibliographicinformation available on this topic. From the completely asymptomatic patient to the child with signs of chronic liver disease, the full spectrum is highly varied, compelling to guide the investigation efficiently, in order to avoid unnecessary costs and suffering to the patient and their family. Coordinated work with the hepatologist is advisable in most instances. Key words: transaminases, hypertransaminasemia, children.}

http:/ /dx.doi.org/10.5546/aap.2014.369

\section{INTRODUCCIÓN}

a. Unidad de Hígado y Trasplante Hepático. Sanatorio de Niños. Rosario, Argentina.

b. Departamento de Pediatría. CHUSainte Justine, Universidad de Montreal. Canadá.

Correspondencia:

Dr. Alejandro

Costaguta: alejandro.

costaguta@gmail.com

Conflicto de intereses: Ninguno que declarar.

Recibido: 14-1-2014 Aceptado: 15-1-2014 trata de niños, lo que pone al pediatra ante un desafío de difícil solución.

En la presente revisión, intentaremos ilustrar, a través de ejemplos clínicos, algunas causas menos conocidas de hipertransaminasemia en el niño a fin de identificar rápidamente las patologías que cuentan con un tratamiento eficaz e indicar la consulta al especialista cuando sea necesario.

\section{Conceptos fisiopatológicos básicos}

Las transaminasas, o aminotransferasas, son enzimas que catalizan la transferencia de un grupo alfa-amino entre aminoácidos y el ácido alfa-cetoglutárico, generando glutamina y el ácido correspondiente. La transaminasa glutámico-oxalacética (TGO) genera ácido oxalacético a partir de aspartato, en tanto que la transaminasa glutámico-pirúvica (TGP) genera ácido pirúvico a partir de alanina. ${ }^{1}$

La TGO, cuya vida media es de $17 \mathrm{~h}$, se distribuye ampliamente en miocardio, hígado, músculo estriado, riñones, páncreas, bazo, pulmones y eritrocitos; en tanto que la TGP, con una vida media de $45 \mathrm{~h}$, está presente sobre todo en hígado y, en menor concentración, músculo esquelético, páncreas, bazo y pulmones. Esta última característica destaca a la TGP como marcadora más específica de hepatopatía.

Es importante recordar esta distribución, ya que no existen isoenzimas específicas de tejido para las aminotransferasas. ${ }^{1,2}$

\section{Evaluación del paciente pediátrico con aumento de transaminasas}

El aumento de transaminasas en el suero es el motivo más frecuente de consulta en hepatología pediátrica. Enfrentados a esta situación, el primer 
paso debería ser la confirmación del resultado previo. Infecciones que podemos denominar habituales y sin consecuencias, medicamentos o hasta productos naturales, o un ejercicio intenso pueden ser responsables de un aumento transitorio de las aminotransferasas séricas. En general, en estos casos, el aumento es mínimo y de breve duración. ${ }^{3}$

El nivel sérico de las enzimas varía en relación con la patología que lo produce. Si bien las cifras muy elevadas (superiores a $1000 \mathrm{UI} / \mathrm{ml}$ ), en general, revelan daño extenso, especialmente cuando predomina la TGO, el dato no es en sí mismo un buen predictor del compromiso en la función hepatocelular. En cambio, un descenso de las transaminasas asociado a un aumento de las cifras de bilirrubina o del tiempo de protrombina es un buen indicador de mal pronóstico, por lo que la evolución de los valores en relación con los otros parámetros es más útil que la valoración de las cifras absolutas como dato aislado. ${ }^{4}$

El aumento de transaminasas puede observarse en forma aislada o asociado a manifestaciones de hepatopatía (ictericia, hepatomegalia, etc.).

Una serie de ejemplos descritos a continuación pueden ilustrar con más claridad este concepto.

\section{AUMENTO AISLADO DE AMINOTRANSFERASAS SÉRICAS \\ Paciente 1}

Niña de 9 años de edad, que consulta por un aumento aislado de transaminasas. En el examen clínico, no se reportan ictericia, signos de hepatopatía crónica ni hepatoesplenomegalia. Los exámenes de laboratorio muestran bilirrubina total y conjugada normales. TGP de $95 \mathrm{UI} / \mathrm{L}$, TGO de 129 UI/L, gamaglutamiltranspeptidasa (GGT) normal, además de albúmina y razón internacional normatizada (RIN) normales.

\section{¿Cuál sería la actitud apropiada?}

Respuesta: asegurarse de que el origen de las aminotransferasas, con un aumento de la TGO mayor que la TGP, no sea de origen muscular. El dosaje de la creatin-kinasa (CPK) debe realizarse antes de continuar la investigación. En este caso, los niveles séricos de CPK fueron de 2,790 UI/L, o sea más de 10 veces los valores normales. La paciente sufre de una miopatía facioescápulodeltoidea.

En un centro de referencia en hepatología pediátrica, recibimos de 3 a 4 veces por año este tipo de paciente, asintomático u oligosintomático desde el punto de vista muscular. Dos series publicadas recientemente muestran una demora significativa entre el momento de los primeros signos de miopatía y el diagnóstico definitivo de la enfermedad. ${ }^{5,6}$ El bajo nivel de sospecha explica por qué nos orientamos en primera instancia (incorrectamente) hacia una hepatopatía.

\section{Paciente 2}

Niña de 3 años, que consulta por aumento de aminotransferasas séricas. No evidencia ningún síntoma o signo relacionado con una enfermedad hepática o muscular. La evaluación bioquímica mostró TGP normal, TGO de 240 UI/L, GGT, albúmina y RIN normales.

\section{¿Cuál es la próxima etapa en la investigación?}

Respuesta: repetir el examen y, si el aumento de TGO se confirma, realizar una electroforesis y precipitación con polietilenglicol (PEG) de la proteína aumentada. Diagnóstico para esta paciente: presencia de macro-TGO en sangre.

Datos recientemente publicados ${ }^{7}$ muestran que casi $40 \%$ de los niños con un aumento aislado de TGO tenían macro-TGO circulantes en el momento del examen bioquímico. Diferentes enzimas circulantes (entre ellas, la TGO) pueden formar, de manera transitoria y por causas desconocidas, complejos de alto peso molecular con otros componentes del plasma o por polimerización consigo misma. Como consecuencia del alto peso molecular, estos macroagregados no son filtrados en el glomérulo renal y persisten en circulación. La presencia de macro-TGO puede persistir por años y desaparecer con el tiempo sin constituir patología alguna. La precipitación de estas macromoléculas con PEG permite el diagnóstico bioquímico de una manera sencilla, lo que evita la realización de costosos exámenes en la exploración de un aumento aislado de TGO sérica.

\section{Paciente 3}

Niño de 3 años de edad, sin antecedentes patológicos personales o familiares; presentó un cuadro de fiebre $\left(38^{\circ} \mathrm{C}\right)$ acompañado de astenia e hiporexia de 3 días de evolución. En un examen de laboratorio, su pediatra constató aumento de la TGO (450 UI/L) y la TGP (670 UI/L), con hemograma y plaquetas normales. A los 10 días del comienzo, el paciente desarrolló una erupción micropapular generalizada y adenopatías cervicales. A pesar del buen estado general (estaba afebril), agregó lesiones purpúricas en miembros inferiores, que duraron 1 semana y desaparecieron. Las transaminasas se mantuvieron elevadas durante toda la evolución 
y se normalizaron al segundo mes. Los padres negaban la ingesta de tóxicos u otros fármacos, excepto ibuprofeno ocasionalmente durante los picos de fiebre. La serología para virus $A, B, C$, EBV, CMV, HHV 6, enterovirus y parvovirus B19 resultó negativa. Un año más tarde, el paciente continúa asintomático y con resultados de laboratorio normales.

Este cuadro descrito corresponde a una elevación inespecífica de transaminasas, como fenómeno asociado a una activación del sistema inmune en respuesta a una noxa desconocida (bystander hepatitis). Ocasionalmente, virus no hepatotropos o enfermedades del colágeno pueden motivar la hipertransaminasemia, que se normaliza cuando se controla la causa original. La etiología obedece al secuestro intrahepático de linfocitos circulantes activados, capaces de producir inflamación y grados variables de necrosis de los hepatocitos vecinos. ${ }^{8}$ Estos procesos, como se dijo, son autolimitados $\mathrm{y}$, dada su evolución benigna, solo requieren controles clínicos y de laboratorio hasta su normalización.

\section{Paciente 4}

Niña de 4 años de edad, asintomática, en control por peso estacionario durante los últimos 3 meses. Se detecta una TGO de $98 \mathrm{UI} / \mathrm{L}$, una TGP de $80 \mathrm{UI} / 1$, sin otras alteraciones en el resto del laboratorio, que incluye hemograma, eritrosedimentación, glucemia, uremia, orina, proteinograma sérico, bilirrubina, GGT, fosfatasa alcalina y urocultivo. Al momento del examen físico, el peso se encontraba en percentil 10, con una talla en percentil 50, distensión abdominal hipertimpánica leve e hígado palpable a $2 \mathrm{~cm}$ del reborde costal, de consistencia normal. Se solicitaron anticuerpos antitransglutaminasa en $\operatorname{Ig} A$ e $\operatorname{Ig} G$, que resultaron positivos a título elevado. Una biopsia endoscópica de la mucosa duodenal reveló atrofia subtotal de las vellosidades, lo que confirmó el diagnóstico de enfermedad celíaca. La niña comenzó una dieta libre de gluten, y se normalizaron los valores de transaminasas a las ocho semanas.

La asociación entre hipertransaminasemia y enfermedad celíaca ha sido reconocida desde hace mucho tiempo. ${ }^{9}$ Un metaanálisis reciente revela que aproximadamente uno de cada tres niños celíacos presenta aumento de transaminasas al momento del diagnóstico. ${ }^{10}$ Este aumento responde con rapidez a la exclusión del gluten ${ }^{11}$ y su origen es probablemente una activación inespecífica del sistema inmune intrahepático frente a un intestino con permeabilidad anormal de antígenos (leaky gut). Con menos frecuencia, el cuadro corresponde a una hepatitis autoinmune definida (alrededor del 1\% de los pacientes celíacos); estos últimos pacientes probablemente representen un subgrupo diferente, con predisposición genética particular, y deben recibir tratamiento inmunosupresor para controlar la hepatopatía. Por último, existen pacientes celíacos con hipertransaminasemia y esteatosis hepática como única expresión, por lo que debería excluirse la enfermedad celíaca en todo niño con aumento de transaminasas, no obeso y con signos de esteatosis en la ecografía o biopsia. ${ }^{10}$

\section{AUMENTO DE AMINOTRANSFERASAS SÉRICAS CON SIGNOS DE ENFERMEDAD HEPÁTICA \\ Paciente 5}

Una niña de 12 años de edad se presenta con cifras elevadas de transaminasas (TGO: 650 UI/L; TGP: $780 \mathrm{UI} / \mathrm{L}$ ) solicitadas para estudiar un cuadro de astenia e hiporexia de 2 meses de evolución. Al momento del examen físico, se constataban numerosos nevus estelares en la cara, el tronco y los miembros superiores, un hígado aumentado de consistencia a predominio del lóbulo izquierdo $(5 \mathrm{~cm})$, con un polo de bazo palpable a $4 \mathrm{~cm}$ del reborde costal. El hemograma no mostraba alteraciones, como tampoco el resto de los parámetros de laboratorio de rutina ni la ecografía abdominal. La serología para virus A, B, C, CMV y EBV era (-). La ceruloplasmina era normal. El proteinograma sérico evidenció cifras elevadas de gammaglobulinas, confirmadas con un valor de IgG de 4,100 mg \%. Los anticuerpos antimúsculo liso fueron $(+)$ a valores de $1 / 650$, con un FAN (+) 1/1250. Con diagnóstico de hepatitis autoinmune tipo 1, confirmado por los hallazgos de la biopsia hepática, se inició un tratamiento inmunosupresor y se alcanzó la remisión en los meses subsiguientes.

La hepatitis autoinmune es un proceso crónico necroinflamatorio progresivo del hígado, de etiología desconocida, caracterizado por una marcada hipergammaglobulinemia y autoanticuerpos circulantes que definen dos tipos diferentes: FAN y antimúsculo liso para el tipo 1, y anti-LKM para el tipo $2 .{ }^{12}$ Esta es una enfermedad predominantemente femenina en proporción de 3-4 a 1, que afecta en particular a la edad prepuberal y adolescente, por lo que es la causa más frecuente de hepatitis crónica en pediatría. La presentación clínica es muy variable, con formas "agudas" caracterizadas por el aumento significativo de transaminasas, 
grados variables de ictericia y hepatomegalia en $30-50 \%$ de los pacientes. En una proporción similar, se observan formas menos evidentes, con síntomas inespecíficos, en los que la sospecha diagnóstica debe estar presente para conducir la investigación. La asociación con otras enfermedades autoinmunes se observa en el 20$30 \%$ de los pacientes o su familia inmediata, y su presencia contribuye a sospechar el diagnóstico. El tratamiento inmunosupresor es efectivo para controlar la enfermedad en más de un $90 \%$ de los casos. ${ }^{13}$

\section{Paciente 6}

Paciente varón, de 16 años de edad, con aumento de enzimas hepáticas descubierto en una investigación de laboratorio por astenia e hiporexia (TGP: 91 UI/L; TGO: 48 UI/L; GGT: $201 \mathrm{UI} / \mathrm{L})$. La ecografía de abdomen no reveló alteraciones, por lo que, ante la persistencia de las anomalías bioquímicas, se procedió a realizar una biopsia hepática, que mostró afectación de los espacios porta con fibrosis concéntrica alrededor de los conductos biliares y confirmó, con una colangio-resonancia magnética, la presencia de una colangitis esclerosante primaria.

La colangitis esclerosante primaria es un proceso inflamatorio obliterativo y destructivo del árbol biliar intra y extrahepático, cuya etiología es desconocida. Se trata de una enfermedad infrecuente, de lenta evolución y difícil diagnóstico, especialmente en las etapas iniciales. En un $80 \%$ de los casos, se asocia a colitis ulcerosa o enfermedad de Crohn y, en menor porcentaje, a síndromes de inmunodeficiencia e histiocitosis. ${ }^{14}$ El diagnóstico se sospecha por la presencia de una hepatopatía crónica, con un aumento significativo de las enzimas de colestasis (GGT y fosfatasa alcalina), muy por encima de la elevación de las transaminasas, y se confirma con la histología y el estudio radiológico del árbol biliar. Actualmente, el estudio por resonancia magnética nuclear es el preferido, por la ausencia de radiaciones y la alta calidad de imágenes obtenidas con los equipos más modernos. Si bien no existe un tratamiento específico, la utilización de ácido ursodesoxicólico puede mejorar los parámetros de laboratorio y retrasar la evolución de la enfermedad.

\section{Paciente 7}

Varón de 6 años de edad, con aumento de enzimas hepáticas, descubierto en forma fortuita (TGO: 96 UI/L; TGP: 174 UI/L; GGT: 224 UI/L). El niño se encontraba en excelente estado general y solo refería prurito nocturno ocasional. La madre relataba colestasis (prurito intenso) durante el embarazo y litiasis biliar con colecistectomía a la edad de 25 años. El examen físico revelaba solo un hígado palpable a $2 \mathrm{~cm}$ del reborde costal, de consistencia normal. La ecografía abdominal y un estudio de la vía biliar por resonancia magnética no mostraron alteraciones. La biopsia hepática evidenciaba colestasis, fibrosis portal incipiente y discreta proliferación colangiolar. El estudio genético reveló la presencia de una mutación en el gen ABCB4, que confirmó el diagnóstico de colestasis familiar progresiva tipo 3 . El tratamiento con ácido ursodesoxicólico normalizó las alteraciones bioquímicas y el prurito.

Las colestasis familiares progresivas son un grupo de patologías producidas por el defecto en alguno de los transportadores del canalículo biliar, con la consecuente alteración del flujo y retención de componentes de la bilis. Las cifras de GGT en suero permiten distinguir dos categorías: normal en el tipo 1 y 2, y elevada en el tipo $3 .{ }^{15}$ En este último, la disfunción se produce en el transportador de fosfolípidos (fosfatidilcolina) que no pueden ser exportados desde el hepatocito, por lo que las sales biliares permanecen libres en suspensión, ejerciendo una acción detergente sobre el epitelio biliar, lo que provoca su destrucción y eventual fibrosis. Es frecuente constatar antecedentes familiares de litiasis biliar en edades precoces y colestasis del embarazo o durante la ingesta de anticonceptivos orales. La administración de ácido ursodesoxicólico detiene el proceso en un porcentaje significativo de pacientes y evita la progresión de las lesiones. ${ }^{16}$

\section{Paciente 8}

Niño de 10 años de edad, con antecedentes de astenia y distensión abdominal de varios meses de evolución. Agregó ictericia y coluria por lo que se le solicitaron exámenes de laboratorio, que revelaron bilirrubina de $18 \mathrm{mg} \%$ (directa $16 \mathrm{mg} \%$ ), TGO de $685 \mathrm{UI} / \mathrm{L}, \mathrm{TGP}$ de $88 \mathrm{UI} / \mathrm{L}$, fosfatasa alcalina de $67 \mathrm{UI} / \mathrm{L}, \mathrm{GGT}$ de $72 \mathrm{UI} / \mathrm{L}$, albúmina sérica de $1,8 \mathrm{~g} \%$, tasa de protrombina de $30 \%$ y gammaglobulinas de $2,1 \mathrm{~g} \%$. El examen físico mostraba un hígado palpable, duro a $3 \mathrm{~cm}$ de reborde costal, y signos de ascitis, sin esplenomegalia. Presentaba ictericia conjuntival y numerosos nevus estelares en el tronco, la cara y los miembros superiores. Los marcadores virales y de autoinmunidad fueron negativos. Los valores de ceruloplasmina sérica estaban 
disminuidos (9 mg/dl); con el dosaje de cobre urinario elevado y la presencia de anillos de Kayser-Fleischer, se confirmó el diagnóstico de enfermedad de Wilson.

La enfermedad de Wilson se caracteriza por una incapacidad para excretar cobre en la bilis, por deficiencia en la proteína transportadora ATP7B. Su presentación clínica es muy variada, por lo que se debe tener presente en el diagnóstico diferencial de cualquier hepatopatía, desde las formas crónicas hasta la presentación fulminante. ${ }^{17}$

Una característica destacable, en este último contexto, es la desproporción que suele existir entre los valores muy elevados de bilirrubina sérica y la fosfatasa alcalina baja. El aumento de las inmunoglobulinas puede ser un elemento de confusión en el diagnóstico diferencial con la hepatitis autoinmune. En ocasiones, la presencia de anemia hemolítica puede orientar el diagnóstico, especialmente en las formas agudas. La disminución de la ceruloplasmina sérica, el aumento de cobre urinario y la presencia de anillos de Kayser-Fleischer en el examen ocular sugieren el diagnóstico, que se confirma con el dosaje de cobre en tejido hepático, en los casos en que se pueda implementar. ${ }^{18}$ Dado que existe un tratamiento efectivo (quelación), deben hacerse todos los esfuerzos para diagnosticar esta enfermedad, que, librada a su evolución natural, muestra un pronóstico ominoso.

\section{CONCLUSIÓN}

Las causas de perturbación de los exámenes hepáticos son múltiples, como lo muestran parcialmente los ejemplos precedentes, resumidos en las Tablas 1 y 2. En ocasiones, la hipertransaminasemia remite espontáneamente en un tiempo variable (meses), sin que pueda establecerse una etiología. ${ }^{3}$ Estos niños solo requieren seguimiento clínico, en conjunto con el hepatólogo.

Es imprescindible asegurar, en primer término, que el aumento de transaminasas obedece a una causa hepática, lo que se corrobora por valores normales de CPK en sangre.

En todas las situaciones, el pediatra debe interrogar a la familia sobre la ingestión de infusiones o preparados caseros, como así también de medicamentos u otras drogas, ya que la única forma de diagnosticar oportunamente una toxicidad hepática es pensar en esa posibilidad. ${ }^{19}$

En muchos casos, el diagnóstico no resulta complicado (enfermedad celíaca, macro-TGO). En otras situaciones, la consulta con el especialista es

\section{TABLA 1. Causas de hipertransaminasemia agrupadas de acuerdo con otros parámetros de laboratorio}

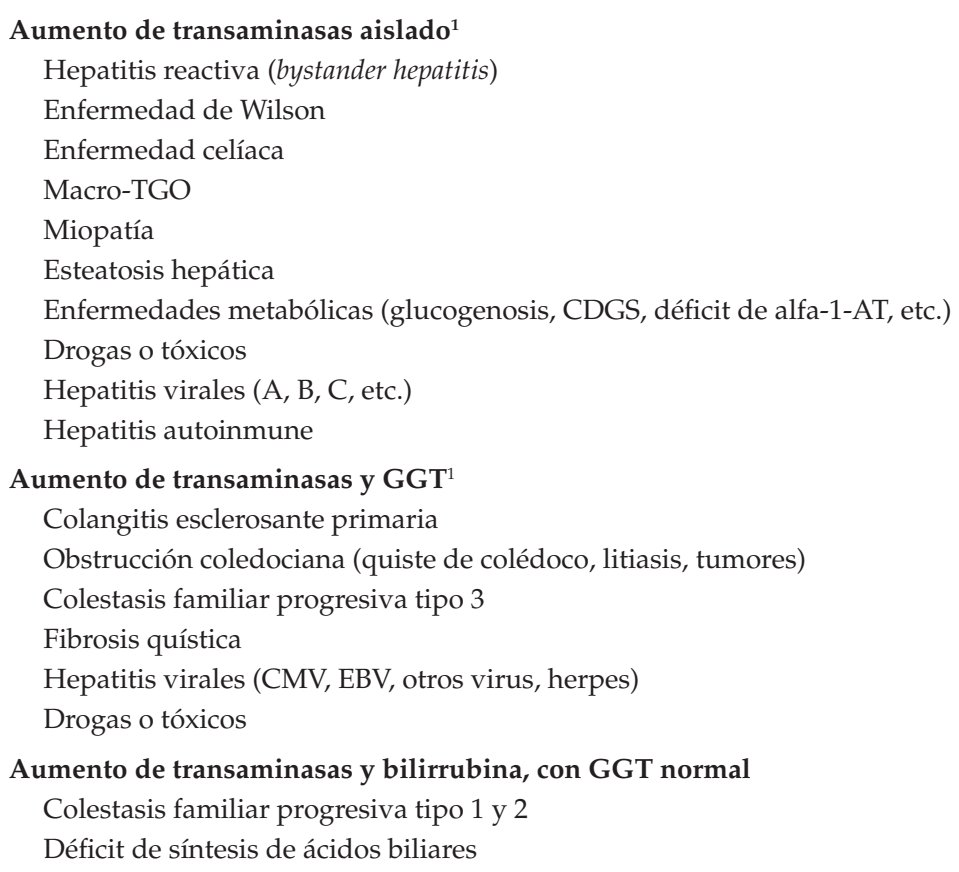

1. Pueden presentar aumento de bilirrubina en alguna etapa de su evolución. 
TABLA 2. Causas de hipertransaminasemia de acuerdo con la forma de presentación y evolución

\begin{tabular}{l} 
Agudas \\
Hepatitis virales: virus A, virus $\mathrm{E}^{1}$ \\
Hepatitis virales (otros): CMV, EBV, enterovirus, parvovirus, HSV ${ }^{1}$ \\
Hepatitis isquémica (hipoxia) ${ }^{1}$ \\
Hepatitis por drogas o tóxicos ${ }^{1}$ \\
Obstrucción biliar \\
Hepatitis autoinmune \\
Enfermedad de Wilson \\
Otras enfermedades metabólicas \\
Crónicas \\
Hepatitis virales: virus B, virus C \\
Esteatosis hepática no alcohólica \\
Hepatitis por drogas o tóxicos \\
Hepatitis autoinmune \\
Colangitis esclerosante \\
Hepatitis reactiva (bystander hepatitis) \\
Enfermedad celíaca \\
Colestasis familiar progresiva \\
Enfermedad de Wilson \\
Déficit de alfa-1-antitripsina (fenotipo Pi-Z) \\
Fibrosis quística \\
Otras enfermedades metabólicas \\
\hline
\end{tabular}

1. Pueden presentar cifras de TGO en suero muy elevadas (> $1000 \mathrm{UI} / \mathrm{L})$.

imprescindible a fin de orientar la investigación de manera rápida y eficiente, y evitar gastos innecesarios y sufrimiento a los pacientes.

\section{BIBLIOGRAFÍA}

1. Burke MD. Liver function: test selection and interpretation of results. Clin Lab Med 2002;22(2):377-90.

2. Rigato I, Ostrow J, Tiribelli C. Biochemical investigation in the management of liver disease. En: Rodés J, Benhamou JP, Blei A, Reichen J, Rizzetto M, eds. Textbook of Hepatology. From basic science to clinical practice. $3^{\text {rd }}$ ed. Oxford: Blackwell Publishing Ltd;2007:451-67.

3. Iorio R, Sepe A, Giannattasio A, Cirillo F, Vegnente A. Hypertransaminasemia in childhood as a marker of genetic liver disorders. J Gastroenterol 2005;40(8):820-6.

4. Frauca Remacha E. Ictericia y/o hipertransaminasemia. Actitud diagnóstico-terapéutica. Pediatr Integral 2003;7(3):187-98.

5. VeropalumboC,DelGiudiceE,Esposito G,MaddalunoSet al. Aminotransferases and muscular diseases: a disregarded lesson. Case reports and review of the literature. J Paediatr Child Health 2012:48(10);886-90.

6. Wright MA, Yang ML, Parsons JA, Westfall JM, Yee AS. Consider muscle disease in children with elevated transaminase. J Am Board Fam Med 2012;25(4):536-40.

7. Caropreso M, Fortunato G, Lenta S, Palmieri D, et al. Prevalence and long-term course of macro-aspartate aminotransferase in children. J Pediatr 2009;154(5):744-8.

8. Alvarez F. The diagnosis and classification of immune mediated hepatic diseases. En: Gershwin M, Vierling J, Manns M, eds. Liver immunology. Principles and practice. $2^{\text {nd }}$ ed. New York: Springer;2014:95-109.

9. Ludvigsson JF, Elfström P, Broomé U, Ekbom A, Montgomery SM. Celiac disease and the risk of liver disease: a general population-based study. Clin Gastroenterol Hepatol 2007;5(1):63-9.

10. Vajro P, Paolella G, Maggiore G, Giordano G. Pediatric celiac disease, cryptogenic hypertransaminasemia, and autoimmune hepatitis. J Pediatr Gastroenterol Nutr 2013;56(6):663-70.

11. Olivan del Cacho M, Ros Mar L. Hipertransaminasemia como primera manifestación de enfermedad celíaca. An Esp Pediatr 1998;48(5):537-8.

12. Alvarez F. Autoimmune hepatitis. En Suchy F, Sokol R, BalistreriW, eds. Liverdisease in children. $2^{\text {nd }}$ ed. Philadelphia: Lippincott Williams \& Wilkins;2001:429-41.

13. Mieli-Vergani G, Heller S, Jara P, Vergani D et al. Autoimmune hepatitis. I Pediatr Gastroenterol Nutr 2009;49(2):158-64.

14. Alvarez F. Autoimmune hepatitis and primary sclerosing cholangitis. Clin Liver Dis 2006;10(1):89-107.

15. Tomer G, Shneider BL. Disorders of bile formation and biliary transport. Gastroenterol Clin North Am 2003;32(3):839-55.

16. Colombo C, Vajro P, Degiorgio D, Coviello D et al. Clinical features and genotype-phenotype correlations in children with progressive familial intrahepatic cholestasis type 3 related to ABCB4 mutations. J Pediatr Gastroenterol Nutr 2011;52(1):73-83.

17. Roberts EA, Schilsky ML. A practice guideline on Wilson disease. Hepatology 2003;37(6):1475-92.

18. Nicastro E, Ranucci G, Vajro P, Vegnente A, Iorio R. Re-evaluation of the diagnostic criteria for Wilson disease in children with mild liver disease. Hepatology 2010;52(6):1948-56.

19. Björnsson ES, Bergmann OM, Björnsson HK, Kvaran $\mathrm{RB}$, Olafsson S. Incidence, presentation, and outcomes in patients with drug-induced liver injury in the general population of Iceland. Gastroenterology 2013;144(7):1419-25. 